\title{
Machine Learning \\ Applied to Low Back \\ Pain Rehabilitation - A \\ Systematic Review
}

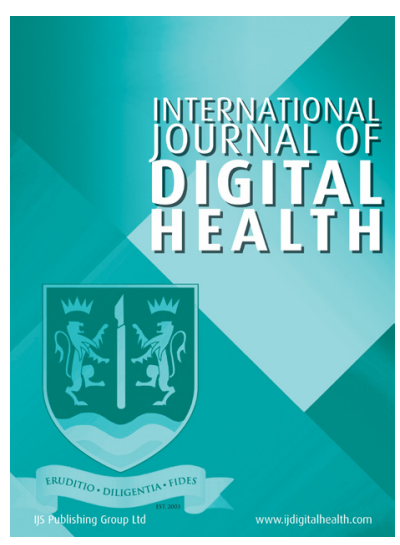

PAULA AMORIM (1)

JOÃO RUIVO PAULO (D)

PAULA ALEXANDRA SILVA (D)

PAULO PEIXOTO (D)

MIGUEL CASTELO-BRANCO (1)

HENRIQUE MARTINS (D)

*Author affiliations can be found in the back matter of this article

\section{ABSTRACT}

Background: Low back pain (LBP) is the leading cause of disability worldwide and an important cause of work absenteeism in the active population. As a recurrent condition, prevention is crucial. Home exercises are effective, but adherence and accurate performance of the exercises are difficult to monitor by doctors and therapists. Machine learning (ML) applied to rehabilitation systems could be a solution to address telerehabilitation for people with chronic LBP if it holds sufficient accuracy in monitoring adherence performance while providing patient guidance. The aim was to search and review studies that have used ML techniques for rehabilitation of people with LBP. To develop an understanding on the outcomes measured, the clinical setting (face-to-face rehabilitation or remote rehabilitation) where interventions took place, and the clinical research methodology that has been used.

Materials and Methods: a systematic review was performed based on research material obtained from literature indexed on MEDLINE, Cochrane Central Register of Controlled Trials (CENTRAL), Scopus, Web of Science and IEEE Xplore databases to locate papers focused on the use of ML applied to rehabilitation of LBP.

Results: after revision of the inclusion and exclusion criteria using the PRISMA methodology, only 14 studies remained for the analysis that is presented as a qualitative synthesis.

Conclusions: ML approaches applied to rehabilitation could help health professionals and LBP patients to manage this condition that affects a significant amount of the active population. ML could be applied to support clinical decisions and to guide patients self-manage their LBP remotely, which makes it a potential telerehabilitation solution. More and better studies, with more participants and following guidelines for best research practice are needed to strengthen the clinical evidence. 


\section{HIGHLIGHTS}

- LBP is the leading cause of disability worldwide

- $\quad$ LBP is a recurrent condition in active population and one of the most common causes of workforce absenteeism, being a significant socio-economic problem

- Home exercises are effective for prevention of LBP

- It is difficult to measure the adherence and the performance accuracy of home exercises

- ML techniques can detect trunk range of motion, muscle recruitment and monitor posture and pain and other LBP-related symptoms that support monitoring and guidance of patient performance in a personalized way

- ML applied to reliable non-invasive monitoring procedures is a promising area to promote self-management of LBP in telerehabilitation scenarios

\section{INTRODUCTION}

Low back pain (LBP) is the leading cause of disability worldwide [1]. The incidence of LBP is highest in the third decade of life, with overall prevalence increasing with age until the person is 60-65 years of age and then LBP gradually declines [2]. LBP is a health and socio-economic problem since LBP affects the active population and is the most common cause of workforce absenteeism, mainly for people with high intensity work compared to sedentary work [3-5]. LBP is a recurrent problem - estimates of recurrence at 1 year range from $24 \%$ to $80 \%$ [2], what makes prevention an opportunity. Approximately $90 \%$ of the patients diagnosed are classified as having "non-specific» LBP, where no clear cause can be found [6] and treatment is directed at reducing pain and its consequences. Management of the condition consists of education and reassurance, analgesic medicines, and non-pharmacological therapies [7]. Physical and psychological treatments, information, and education programmes, combined with manual treatments, are likely to be cost-effective options for LBP [8]. The current evidence suggests that exercise alone or in combination with education, is effective in preventing LBP [9]. Home-based rehabilitation programmes are effective in preventing LBP $[10,11]$, but the success of an exercise program depends on the adherence of patients to the treatment plan and on the accurate performance of the exercises, which is problematic to measure accurately [12].

Telerehabilitation (TR) is a solution that allows for home-based rehabilitation. TR research started about 20 years ago [13] with a focus on information technologies to provide remote support, assessment and information to people with physical and/or cognitive impairments [14]. The technology used in TR may include video conferencing platforms, wearable devices, apps, audio and video communication and social media, as well as many research-driven prototypes housed on a variety of platforms [15]. More complex solutions may further incorporate robots [16] and machine-learning-based systems [17].

Machine Learning $(M L)$ is a branch of artificial intelligence that addresses the question of how to build computers that improve automatically through experience [18, 19]. It provides computational methods for accumulating, changing, and updating knowledge-based models in intelligent systems. It provides learning mechanisms which can infer predictions from examples or data.

ML methods are useful in cases where algorithmic solutions are not available, there is a lack of formal models, or the knowledge about the application domain is poorly defined. During the last couple of decades, ML has been increasingly explored in the context of the medical sciences. In this context, ML is being used for the analysis of the importance of clinical parameters and their combinations for prognosis, e.g. prediction of disease progression, for the extraction of medical knowledge for outcomes research, for therapy planning and support, and for overall patient management [20]. There are two main forms of ML, particularly when it comes to classification methods: supervised and unsupervised. In supervised $\mathrm{ML}$, algorithms are given labelled training data, which is analysed for features important to the discrimination of classes, and then «trained» with this data before being tested with unseen unlabelled data. Unsupervised ML is used to identify patterns without prior knowledge of their classes; common forms are cluster analysis (where data is grouped by patterns of characteristics) or association (where rules are discovered by which data is governed) [19].

The majority of publications on AI in spinal disorders are in the domain of diagnosis, followed by prognosis, prediction, and biomechanical for spinal applications [21]. A recent review concluded that AI and particularly $\mathrm{ML}$ could enhance the ability to detect patterns of clinical characteristics in LBP and guide treatment [22]. As there has been enormous progress in the fields of 
artificial intelligence (AI) and data sciences in the last decade, and as there is an increasing development in the Telerehabilitation field, one of the applications of AI in Telerehabilitation is through virtual coaching. A virtual coach can provide guidance and training to users through a set of tasks, with the aim of supporting positive actions or assisting in learning new skills [23]. In LPB virtual coaches could help users to define and preserve an exercise program, suggest problem-solving skills training, or advise patients.

Reliable non-invasive monitoring procedures combined with self-management are crucial to provide the necessary clinical data for reliable TR. Data collected via inertial sensors in wearable technologies can be used to classify whether users are accurately performing and adhering to the exercise, with up to $99.4 \%$ classification accuracy, as demonstrated in [24]. However, this type of approach introduces discomfort to the user, as the sensors must be placed in contact with the body during capture. Furthermore, the sensors must be placed in the exact corresponding positions, a task that takes time and requires experience. The ideal solution would be based on marker less visual perception of the user's posture and position in space, whether static or dynamic. Such a solution, would make it possible for the patient to perform rehabilitation exercises without being restricted to a specific point in the room/scenario nor unconstrained by wearable apparatus, resulting in greater natural movement and effective comfort for the user.

Another challenge in TR is engagement. Several studies suggest that increases in engagement with rehabilitation exercise can result from gamification, by leveraging principals of game design such as meaningful play, feedback, goals, reward, challenge, difficulty, failure and flow, $[25,26]$.

The aim of this systematic review is to identify studies where machine learning techniques were applied for rehabilitation of chronic (defined as pain that continues for 12 weeks or longer) and nonspecific LBP (no clear tissue cause of pain can be found). The research question for this systematic review, which followed the PICOS components (Population, Intervention, Comparison, Outcomes, Study Design) [27] is: in people with chronic and nonspecific LBP (P) what is the utility and effectiveness/ efficacy of Machine Learning applied for Rehabilitation (I) compared with standard/usual care (C), what outcomes (clinical and non-clinical, e.g. acceptability, usability, and adherence) were measured (O) and what types of studies have been done (S).

\section{METHODS}

\subsection{LITERATURE SEARCH}

This review is based on research material obtained from MEDLINE, Cochrane Central Register of Controlled Trials
(CENTRAL), Scopus, Web of Science and IEEE Xplore until January 19, 2021. The selection of articles was performed manually, as described in the following section. No automatic limits were applied. The search terms were those found in the U.S. National Library of Medicine's controlled vocabulary (MeSH - medical subject headings). A search in PROSPERO was made to exclude any revision already initiated. The same search terms were used for all databases to guarantee comparison among obtained results. The search string and Boolean operators were: («machine learning» OR «artificial intelligence») AND (rehabilitation OR physiotherapy OR exercises) AND («back pain» $O R$ «low back pain» $O R$ «lumbar pain»). The review was conducted and reported in accordance with the Preferred Reporting Items for Systematic Reviews and Meta-Analysis (PRISMA) statement [28] (Figure 1). Additional searching included visually scanning reference lists from relevant studies, hand searching key journals and conference proceedings, contacting study authors, experts and other organizations, searching internet resources and citation searching.

\subsection{DATA EXTRACTION}

After removing duplicates, two authors independently screened records based on titles and abstracts of papers. The inclusion criteria were: 1) focus on chronic and non-specific LBP; 2) general population (specific groups such as a particular profession nor practitioners of a specific sport or activity excluded); 3) using ML techniques; 4) focus on clinical outcomes on physical examination (although other outcomes such as usability, acceptability/adherence are also relevant); 5) applicable to rehabilitation settings; 6) human research. No restrictions were included based on race, sex or age. For $\mathrm{ML}$ approaches in LBP, there was no restriction on study design, to ensure that all research on this matter to date was identified. Exclusion criteria were: 1) no peer review of full conference abstract (proceedings excluded); 2) non original research (reviews or opinion papers excluded); 3) not written in English; 4) non published results (e.g. trials in process); 5) non patient involvement research. A record was excluded when marked by the two authors. All the remaining articles were further examined through their full text for exclusion of irrelevant studies. Disagreements were resolved through team discussion. The protocol was registered in PROSPERO (CRD42021232769).

\section{RESULTS}

\subsection{SCREENING RESULTS}

The initial search retrieved 83 records initially. After a stepwise process of screening titles, abstract, and then full-text articles using the defined eligibility criteria, the authors identified 14 studies to be included (see Figure 1). An overview of the articles and their main characteristics can be found in Table 1. 


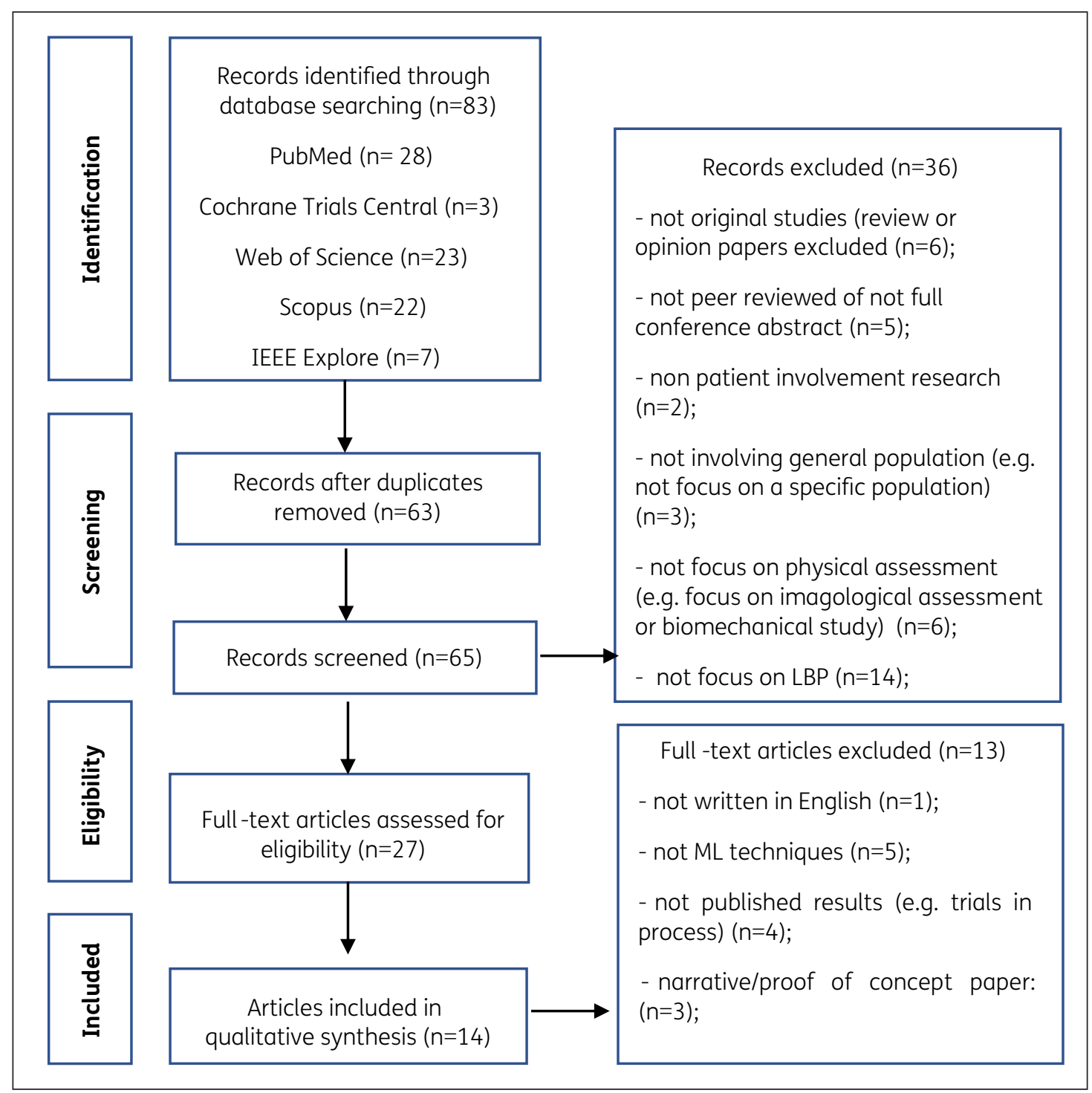

Figure 1 Flow diagram adapted from PRISMA, showing the process for identification and screening of articles for inclusion and exclusion.

\subsection{CHARACTERISTICS OF STUDIES INCLUDED}

Table 1 summarizes the main characteristics of the studies based on PICOS assessment and main studies results. No randomized control trials (RCT) were found. The majority of the studies did not report details on the study methodology used, which prevents quality analysis of the studies. Information on inclusion and exclusion criteria were absent in almost all the studies. Ten studies [30, 32-36, 38, 39, 41, 42] made an effort to have a control group but definition of controls was not always the same: in four studies control group included healthy patients [30, 32, 34, 42], one used datasets from healthy persons [35]; in one study [33] the control group was represented by LBP surgical patients; in three studies [36, 38, 41] the comparison used was experts suggestions/ classification/evaluation (versus ML system suggestions); in one study [34] the algorithm results were compared against a back screening tool results; in one study [39] the results from active LBP patients were compared with healthy controls and with remission LBP patients.
In all studies, the results from $\mathrm{ML}$ solutions were superior to conventional approaches, but that measurement was not always rigorously ascertained and statistically analysed.

The outcomes measured were very heterogeneous. Pain (visual analogue scale) was the main outcome chosen by researchers. Patient questionnaires focusing on disability impact were used in five of the studies [29-31, 33] [38]. Usability, acceptability and adherence were also recorded in two studies [29, 36]. Accuracy in the difference between ML results and experts' opinion/ evaluation was another important concern found in this review $[36,38,41]$ and the results were quite promising.

$\mathrm{ML}$ techniques were also heterogeneous. They used input data from patient questionnaires and from sEMG and motion capture systems. They were used mainly in a supervised $M L$ approach for applications of pain, diagnostics, and exercise prescription. A great number of works rely on Support Vector Machines and Random Forests, which are well-established and well-proven 


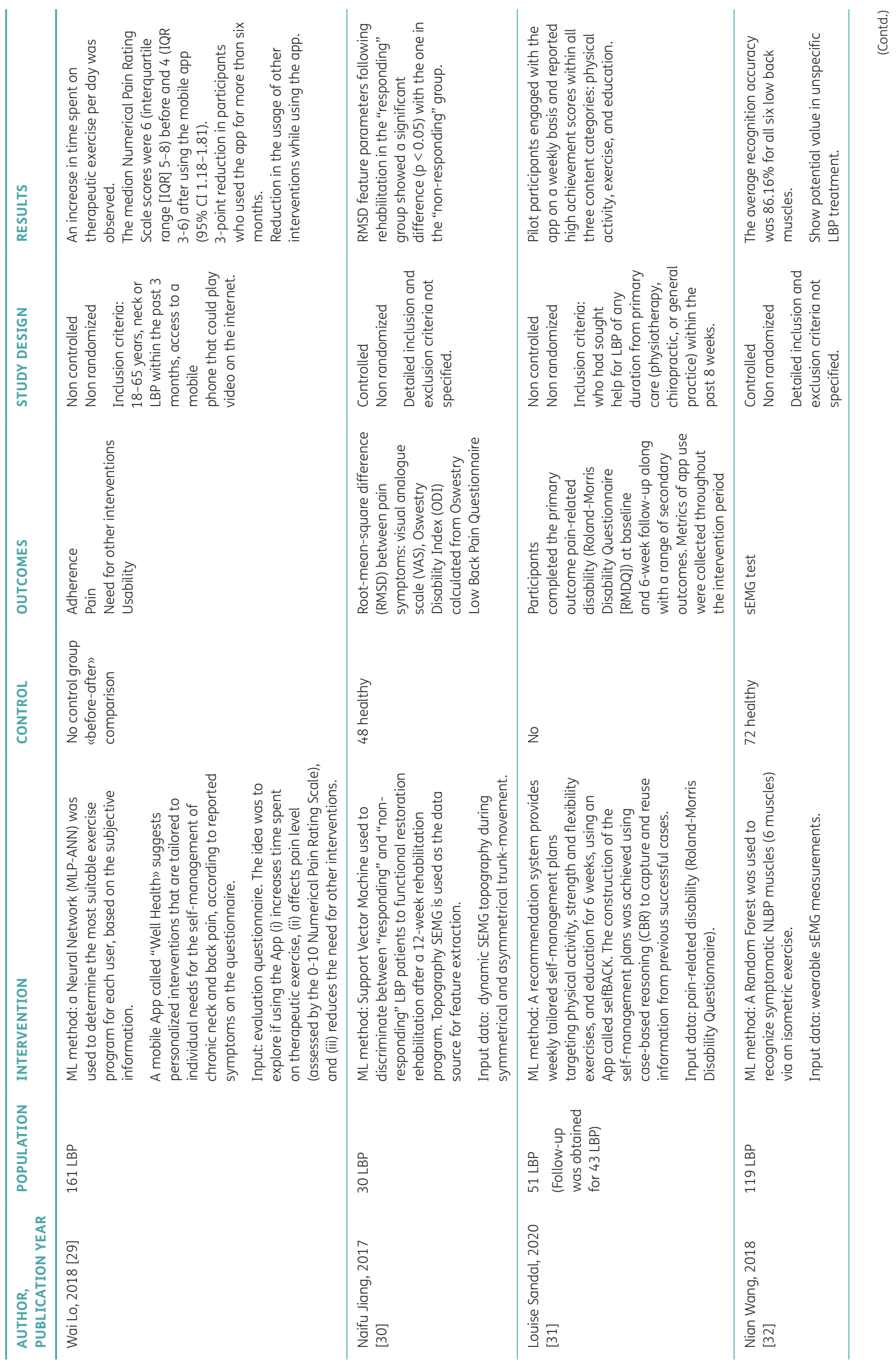




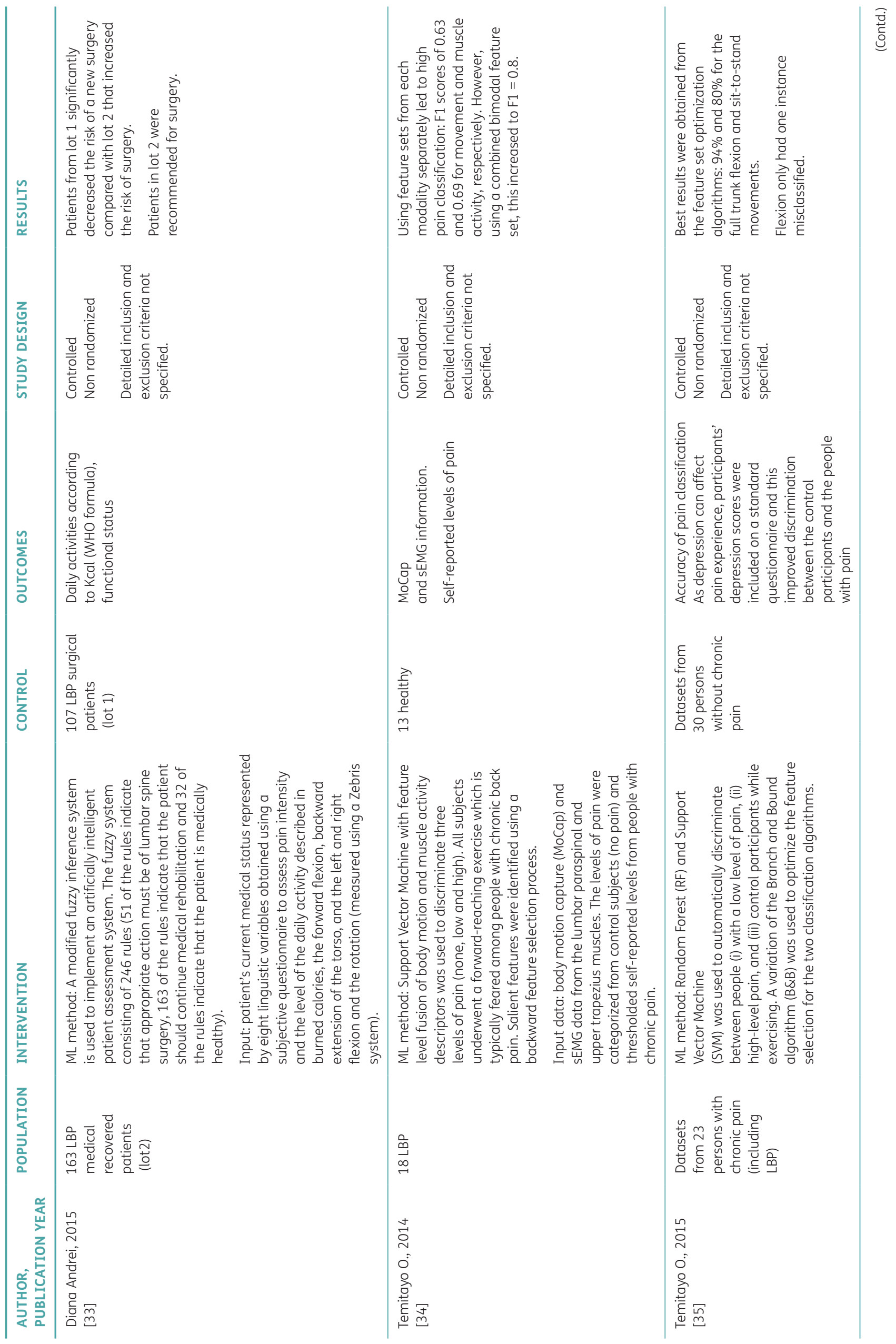




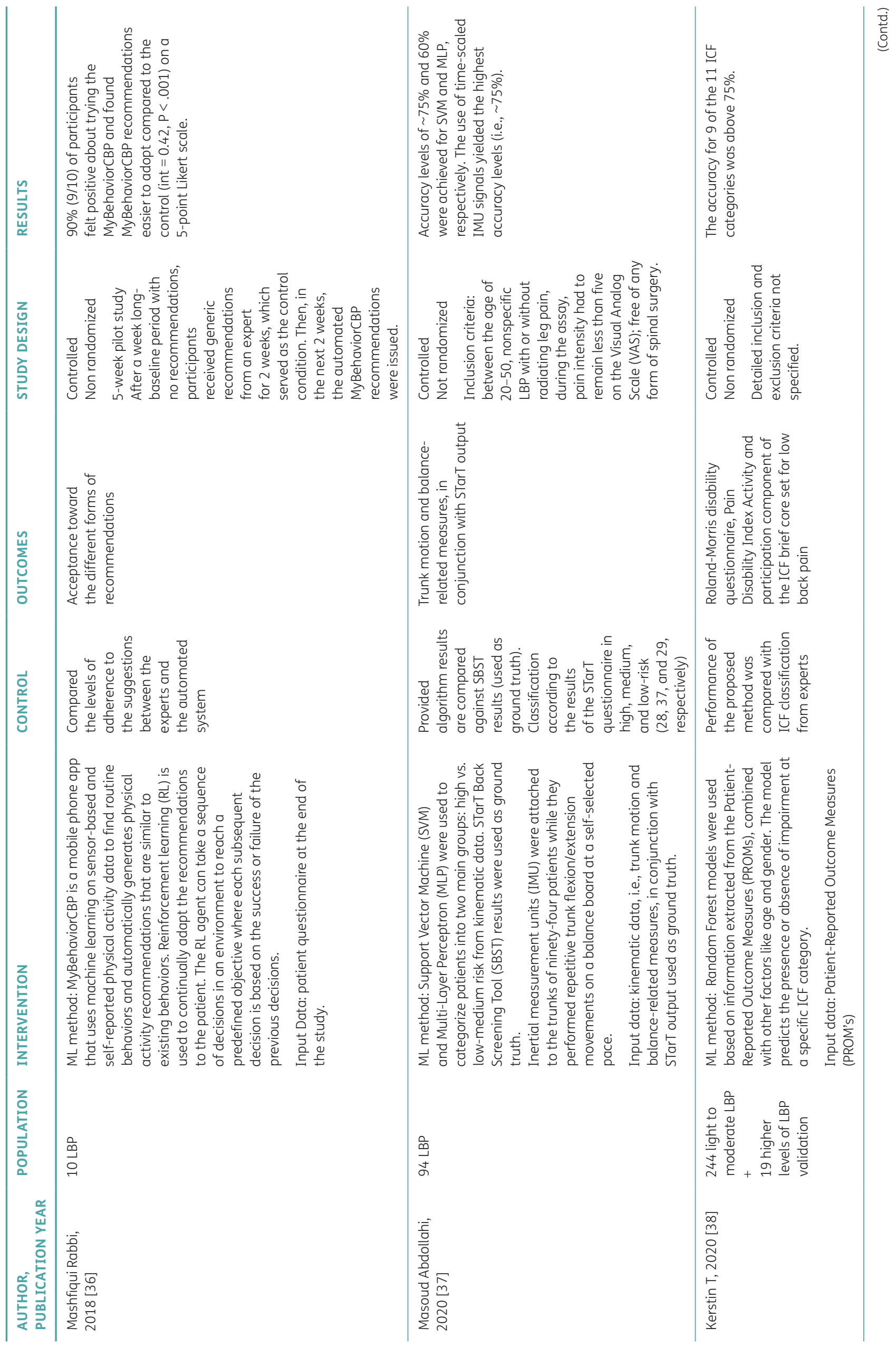




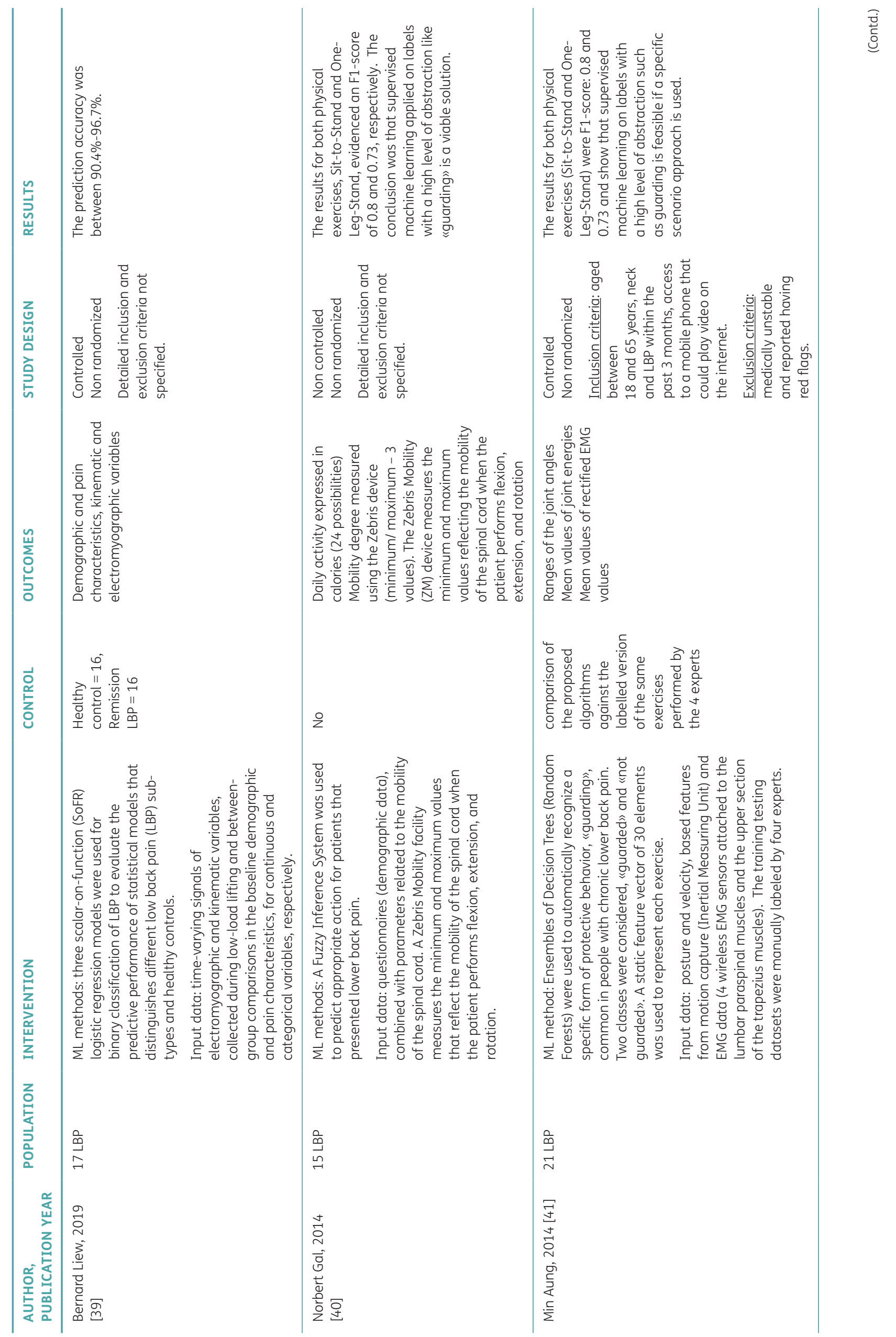




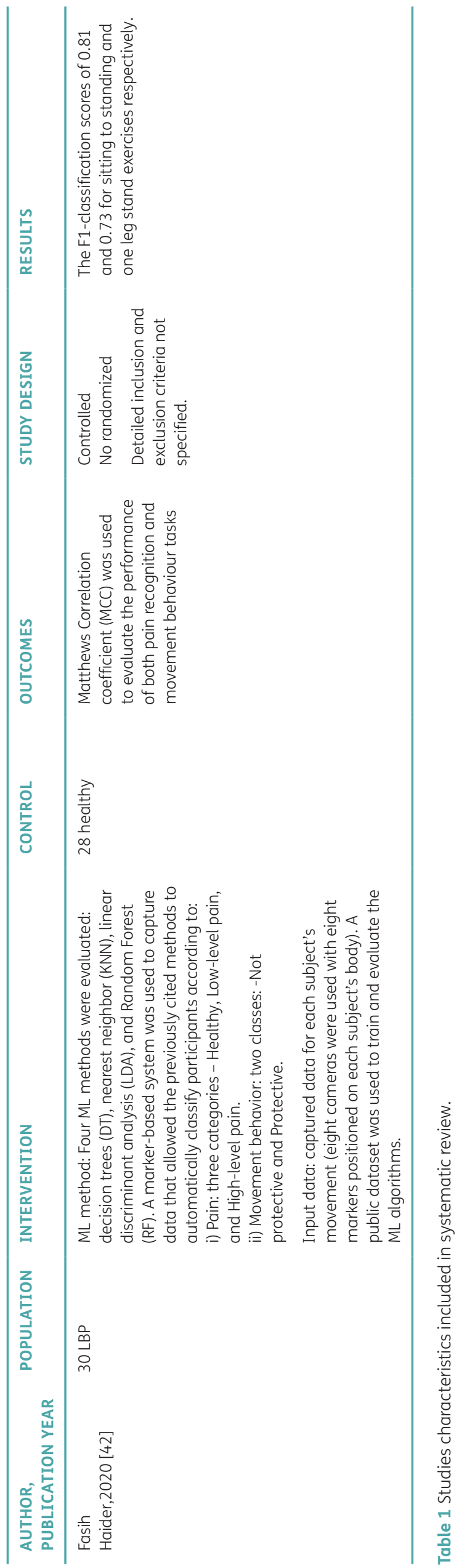


methods in the literature. However, it was disappointing not to find works using more recent approaches used in $\mathrm{ML}$, such as Deep Learning methods, which have shown to be superior to those present in the analysed works. This could be justified by the great demand for big amounts of data, in order for these methods to properly model the problem, which is a constraint for a quick development and deployment strategy.

\section{DISCUSSION}

LBP conventional rehabilitation is based on clinical assessment through information that it is given by patients (such as level of pain or impact in daily living activities) and physical examination (such as posture, muscle contractions, range of motion, gait pattern). Based on that information, doctors decide on the most appropriate therapeutic plan, including ergonomic education, exercises, and medication. ML could help either in the diagnosis [32, 34, 35, 37-39, 41, 42] or in supporting doctors' decisions $[33,40]$ or guiding patients through a personalized therapeutic plan [29, 31, 36], with accuracy and in real-time, giving important clinical information to doctors and therapists and allowing adherence monitoring. ML could also be useful to identify patients' response to functional rehabilitation [30], automatic recognition of fear-avoidance behaviour $[41,42]$ or to categorize patients into different subgroups of LBP risk [37]. Some of the studies using ML for LBP treatment use apps [29, 31, 36], since almost everyone has a smartphone nowadays, including medical students and doctors use medical smartphone apps related to procedure documentation, disease diagnosis, clinical score and drug reference [43]. Pilot studies of mobile phone based personalized physical activity recommendations for chronic pain self-management have shown positive results in feasibility and acceptability by patients [44]. The development of mobile phone apps using Cognitive Behavioural Therapy (CBT) principles is increasing within the research area, in order to increase motivation [45].

The subjective information given by patients is crucial to assess levels of pain or depression. ML cannot substitute the absence of this crucial feedback, but it can help process and analyse such information if it is made available.

Pain assessment is probably the most common feature of LBP for the negative impact on functionality and well-being, being one of the most important determinants in quality of life. Because exercises increase the level of pain, anxiety about anticipated pain increase may lead to seatback and intensified sensitivity to pain [35] and a fear-avoidance behaviour [46]; the avoidance is expressed through self-protective body movement to avoid strain in the painful area, decreasing exercise adherence [41, 42]. Visual Analogue Scale or Oswestry Low Back Pain Questionnaire are examples of most used tools for pain assessment and disability in LBP patients $[30,37]$. Depression scales are also used in LBP research [35], as well as daily activity questionnaires [33, 40]. A more holistic approach is the one given by International Classification of Functioning, Disability and Health (ICF), a standardized reference system for classifying health by accounting for functioning [47]. ML techniques could be applied based on a translation algorithm that automatically links health information reported from LBP patients to categories representing the Activity and Participation components of ICF [38]. Although pain is usually reported by the patient, studies have been done which apply ML based on body motion and muscle activity to discriminate different levels of pain [35]. Other sensing modalities could be used for clinical assessment, such as sEMG (surface electromyography). LBP is related to localized muscle fatigue [48]. There is a significant difference in the patterns of muscles firing phase between symptomatic and healthy muscles, resulting in different muscle compensation in order to reduce the load on a painful tissue to protect from further pain [49]. Based on that fact, some studies used $\mathrm{ML}$ techniques to accurately locate the symptomatic muscles after collecting SEMG [32]. ML can also be used in the development of an assessment system based on a modified fuzzy inference system consisting in a determined number of rules, which could be used to support treatment decisions [33, 40].

A broad search in five databases following a PRISMA checklist in order to find relevant literature on the subject and assess different aspects, was systematically conducted, while recognising some limitations. The main focus was placed on clinical research in setting using machine learning for LBP rehabilitation and not on technical issues or proof of concept papers, which excluded many engineering papers. The inclusion of only articles written in English may contribute to a publication bias. Five databases were included but more databases should be included in future research. Few clinical studies were found and most of them were exploratory studies, with small sample numbers. None of the located studies was a RCT study, which is the "gold standard" for experimental study design standard of clinical research. A fact perhaps related with the recent application of $\mathrm{ML}$ in the Rehabilitation field. The Cochrane seven domains of risk of bias assessment [50], used in randomized trials, were not possible to check due to missing data. For non-randomized studies there are other tools for assessing quality, such as the ROBINS-I [51], but most of the studies were focused on ML solutions feasibility and not in studies quality, making it difficult to apply them. The heterogeneity of studies did not allow a statistical analysis and a rigorous comparison between them. 
It was clear that the focus of the studies was on presenting new solutions and comparing the results of novel approaches with conventional assessment and not on the quality of the clinical research and study design. This is common in the early stages of new technical solutions, where feasibility and pilot studies gradually move towards higher quality studies, in which RCT are included [52]. In clinical research of medical devices, RCTs, and especially ensuring blindness for the participants, is not always easy nor feasible as [53] has highlighted. Nonetheless researchers should strive towards running better quality clinical studies with less bias and hopefully this paper can help them as a steppingstone in this fascinating interface between AI and the human body.

\section{CONCLUSIONS}

Based on the scientific literature, it seems that supervised machine learning approaches applied in rehabilitation could help health professionals and LBP patients to manage this condition that affects a significant percentage of the active population. This could contribute to improving the quality of life of people with LBP and to decrease in work absenteeism, that could ultimately represent economic and social gains. $\mathrm{ML}$ is a promising tool for personalized rehabilitation as it contributes to a new paradigm of healthcare in which interventions are based on individual patient characteristics. ML applied to support clinical decisions has been explored. Some studies used mobile phones apps to help patients selfmanage their LBP remotely, by giving them education material and exercises guides and by monitoring the performance and adherence, being a promising method to use in the telerehabilitation field.

Although all the studies included had better results than conventional approaches, and therefore, provide indicate $M L$ to be a useful tool for the future of LBP rehabilitation, the small number of studies, their heterogeneity in methodology, in outcome measurements, and in quality (for instance no RCT was found), leave to the investigators the opportunity and responsibility to pursue the path towards the strengthening of evidence. A multidisciplinary approach on research in artificial intelligence applied in Health is very useful, as it gives a holistic view and accelerates innovation, but future clinical investigation should be more rigorous. "More and better-quality studies, involving larger samples, using a control group (ideally RCT), and which methodology is rigorously described and follows the guidelines and best practices in research are needed in the future". Engagement is an important issue to be considered in assessing home rehabilitation programmes adherence and this should also be further investigated in future studies. What will make the AI solutions acceptable for clinical use depends on, but is not limited to, the evidence of how the sensors/cameras capture the motions reliably, how the recommendations from ML are clinically relevant/appropriate, and the ease of use of incorporating the $\mathrm{ML}$ recommendations into the exercise. These issues should be kept in mind for future studies.

\author{
ABBREVIATIONS \\ AI - Artificial Intelligence \\ CBT - Cognitive Behavioural Therapy \\ ICF - International Classification of Functioning, Disability \\ and Health \\ LBP - Low Back Pain \\ $M L$ - Machine Learning \\ RCT - Randomized Controlled Trials \\ sEMG - Surface Electromyography \\ TR - Telerehabilitation
}

\section{DATA ACCESSIBILITY STATEMENT}

The databases generated during the current study are available from the corresponding author on reasonable request.

\section{ADDITIONAL FILE}

The additional file for this article can be found as follows:

- Supplement File. AMSTAR 2 Results. DOI: https://doi. org/10.29337/ijdh.34.s1

\section{COMPETING INTERESTS}

The authors have no competing interests to declare.

\section{AUTHOR CONTRIBUTIONS}

The search equation was proposed by the first author and debated with the co-authors until a consensus was reached. The first author, Paula Amorim, has made substantial contributions to the conception, design of the work, acquisition of data, analysis and interpretation of data and has drafted the work. The second author, João Ruivo Paulo, the third author, Paula Alexandra Silva, and fourth author, Paulo Peixoto have made substantial contributions to the drafting, analysis and interpretation of data. The other authors, Miguel Castelo-Branco and Henrique Martins, have made substantial contributions to revising it critically for important intellectual content and final approval of the version to be submitted, identification of limitations and conclusions. 


\section{AUTHOR AFFILIATIONS}

Paula Amorim (D) orcid.org/0000-0003-4908-5642 University of Beira Interior, Faculty of Health Sciences, Covilhã, Portugal; Rehabilitation Medicine Center of Central Region, Portugal

João Ruivo Paulo (D) orcid.org/0000-0003-2924-1687 University of Coimbra, Institute of Systems and Robotics, Department of Electrical and Computer Engineering, Coimbra, Portugal

Paula Alexandra Silva (D) orcid.org/0000-0003-1573-7446 University of Coimbra, Centre for Informatics and Systems of the University of Coimbra, Department of Informatics Engineering, Coimbra, Portugal

Paulo Peixoto (iD) orcid.org/0000-0002-3680-564X

University of Coimbra, Institute of Systems and Robotics, Department of Electrical and Computer Engineering, Coimbra, Portugal

Miguel Castelo-Branco (D) orcid.org/0000-0002-6191-5912 University of Beira Interior, Faculty of Health Sciences, Covilhã, Portugal

Henrique Martins (iD) orcid.org/0000-0001-7535-5103 University of Beira Interior, Faculty of Health Sciences, Covilhã, Portugal

\section{REFERENCES}

1. Vos T, et al. Years lived with disability (YLDs) for 1160 sequelae of 289 diseases and injuries 1990-2010: a systematic analysis for the Global Burden of Disease Study 2010 (in eng). Lancet. Dec 15 2012; 380(9859): 2163-96. DOI: https://doi.org/10.1016/S0140-6736(12)61729-2

2. Hoy D, Brooks P, Blyth F, Buchbinder R. The Epidemiology of low back pain (in eng). Best Pract Res Clin Rheumatol. Dec 2010; 24(6): 769-81. DOI: https://doi.org/10.1016/j. berh.2010.10.002

3. Serranheira F, Sousa-Uva M, Heranz F, Kovacs F, Sousa-Uva A. Low Back Pain (LBP), work and absenteeism. Work. 2020; 65: 63-469. DOI: https://doi.org/10.3233/WOR203073

4. Karunanayake AL, Pathmeswaran A, Kasturiratne A, Wijeyaratne LS. Risk factors for chronic low back pain in a sample of suburban Sri Lankan adult males (in eng). Int. J. Rheum. Dis. Apr 2013; 16(2): 203-10. DOI: https://doi. org/10.1111/1756-185X.12060

5. Metgud DC, Khatri S, Mokashi MG, Saha PN. An ergonomic study of women workers in a woolen textile factory for identification of health-related problems (in eng). Indian J. Occup. Environ. Med. Apr 2008; 12(1): 14-9. DOI: https://doi. org/10.4103/0019-5278.40810

6. Bardin LD, King P, Maher CG. Diagnostic triage for low back pain: a practical approach for primary care (in eng). Med. J. Aust. Apr 3 2017; 206(6): 268-273. DOI: https://doi. org/10.5694/mja16.00828

7. Maher C, Underwood M, Buchbinder R. Non-specific low back pain (in eng). Lancet. Feb 18 2017; 389(10070): 736 747. DOI: https://doi.org/10.1016/S0140-6736(16)30970-9
8. Andronis L, Kinghorn P, Qiao S, Whitehurst DG, Durrell S, McLeod H. Cost-Effectiveness of Non-Invasive and Non-Pharmacological Interventions for Low Back Pain: a Systematic Literature Review (in eng). Appl Health Econ Health Policy. Apr 2017; 15(2): 173-201. DOI: https://doi. org/10.1007/s40258-016-0268-8

9. Steffens D, et al. Prevention of Low Back Pain: A Systematic Review and Meta-analysis (in eng). JAMA Intern Med. 2016; 176(2): 199-208. DOI: https://doi.org/10.1001/ jamainternmed.2015.7431

10. Ben Salah Frih Z, Fendri Y, Jellad A, Boudoukhane S, Rejeb N. Efficacy and treatment compliance of a homebased rehabilitation programme for chronic low back pain: a randomized, controlled study (in eng fre). Ann. Phys. Rehabil. Med. Jul 2009; 52(6): 485-96. DOI: https://doi. org/10.1016/j.rehab.2009.04.002

11. Garcia AN, et al. Effectiveness of back school versus McKenzie exercises in patients with chronic nonspecific low back pain: a randomized controlled trial (in eng). Phys. Ther. Jun 2013; 93(6): 729-47. DOI: https://doi.org/10.2522/ ptj.20120414

12. Peek K, Carey M, Mackenzie L, Sanson-Fisher R. Patient adherence to an exercise program for chronic low back pain measured by patient-report, physiotherapist-perception and observational data (in eng). Physiother. Theory Pract. Dec 2019; 35(12): 1304-1313. DOI: https://doi.org/10.1080/ 09593985.2018 .1474402

13. Jafni TI, Bahari M, Ismail W, Radman A. Understanding the Implementation of Telerehabilitation at PreImplementation Stage: A Systematic Literature Review. Procedia Computer Science. 2017/01/01/2017; 124: 452460. DOI: https://doi.org/10.1016/j.procs.2017.12.177

14. Schwamm LH, Holloway RG, et al. A review of the evidence for the use of telemedicine within stroke systems of care: a scientific statement from the American Heart Association/American Stroke Association (in eng). Stroke. 2009; 40(7). DOI: https://doi.org/10.1161/ STROKEAHA.109.192360

15. Adamovich SV, Fluet GG, Tunik E, Merians AS. Sensorimotor training in virtual reality: a review. (in eng), NeuroRehabilitation. 2009; 25(1): 29-44. DOI: https://doi. org/10.3233/NRE-2009-0497

16. Garrett B, Taverner T, Gromala D, Tao G, Cordingley E, Sun C. Virtual Reality Clinical Research: Promises and Challenges (in eng). JMIR Serious Games. 2018; 6(4): e10839-e10839. DOI: https://doi.org/10.2196/10839

17. Lee M, Siewiorek D, Smailagic A, Bernardino A, Bermúdez i Badia S. Interactive hybrid approach to combine machine and human intelligence for personalized rehabilitation assessment. 2020. pp. 160-169. DOI: https://doi. org/10.1145/3368555.3384452

18. Mitchell T. Machine Learning. McGraw Hill; 1997.

19. Tack C. Artificial intelligence and machine learning | applications in musculoskeletal physiotherapy (in eng). Musculoskelet Sci Pract. Feb 2019; 39: 164-169. DOI: https:// doi.org/10.1016/j.msksp.2018.11.012 
20. Magoulas GD, Prentza A. Machine learning in medical applications. In Machine Learning and Its Applications: advanced lectures: Springer-Verlag; 2001. pp. 300-307. DOI: https://doi.org/10.1007/3-540-44673-7_19

21 Azimi P, et al. A Review on the Use of Artificial Intelligence in Spinal Diseases (in eng). Asian Spine J. 2020; 14(4): 543571. DOI: https://doi.org/10.31616/asj.2020.0147

22. Tagliaferri SD, et al. Artificial intelligence to improve back pain outcomes and lessons learnt from clinical classification approaches: three systematic reviews (in eng). NPJ Digit Med. 2020; 3: 93. DOI: https://doi.org/10.1038/ s41746-020-0303-X

23. Tropea P, et al. Rehabilitation, the Great Absentee of Virtual Coaching in Medical Care: Scoping Review (in eng). J. Med. Internet Res. Oct 1 2019; 21(10): e12805. DOI: https://doi. org/10.2196/12805

24. Burns DM, Leung N, Hardisty M, Whyne CM, Henry P, McLachlin S. Shoulder physiotherapy exercise recognition: machine learning the inertial signals from a smartwatch (in eng). Physiol. Meas. Jul 23 2018; 39(7): 075007. DOI: https://doi.org/10.1088/1361-6579/aacfd9

25. Rizzo A, Kim G. A SWOT Analysis of the Field of Virtual Rehabilitation and Therapy. Presence. 01/01 2005; 14: 119-146. DOI: https://doi.org/10.1162/1054746053967094

26. Barak Ventura R, Nakayama S, Raghavan $P$, Nov 0 , Porfiri $\mathbf{M}$. The Role of Social Interactions in Motor Performance: Feasibility Study Toward Enhanced Motivation in Telerehabilitation (in eng). J. Med. Internet Res. 2019; 21(5): e12708-e12708. DOI: https://doi.org/10.2196/12708

27. Saaiq M, Ashraf B. Modifying "Pico" Question into "Picos" Model for More Robust and Reproducible Presentation of the Methodology Employed in A Scientific Study (in eng). World J Plast Surg. 2017; 6(3): 390-392. [Online]. Available: https://www.ncbi.nlm.nih.gov/pmc/articles/PMC5714990/.

28. Moher D, Liberati A, Tetzlaff J, Altman DG. Preferred reporting items for systematic reviews and meta-analyses: the PRISMA statement (in eng). PLoS Med. Jul 21 2009; 6(7): e1000097. DOI: https://doi.org/10.1371/journal. pmed.1000097

29. Lo WLA, Lei D, Li L, Huang DF, Tong KF. The Perceived Benefits of an Artificial Intelligence-Embedded Mobile App Implementing Evidence-Based Guidelines for the SelfManagement of Chronic Neck and Back Pain: Observational Study (in eng). JMIR Mhealth Uhealth. Nov 26 2018; 6(11): e198. DOI: https://doi.org/10.2196/mhealth.8127

30. Jiang N, Luk KD, Hu Y. A Machine Learning-based Surface Electromyography Topography Evaluation for Prognostic Prediction of Functional Restoration Rehabilitation in Chronic Low Back Pain (in eng). Spine (Phila Pa 1976). Nov 1 2017; 42(21): 1635-1642. DOI: https://doi.org/10.1097/ BRS.0000000000002159

31. Sandal LF et al. A digital decision support system (selfBACK) for improved self-management of low back pain: a pilot study with 6-week follow-up (in eng). Pilot Feasibility Stud. 2020; 6: 72. DOI: https://doi.org/10.1186/s40814-02000604-2
32 Wang N, Xiao J, Cui L. EasiSMR: Recognizing Non-Specific Low Back Pain Symptomatic Muscles Using Multi-Muscles Fusion based Machine Learning. In 2018 IEEE 4th International Conference on Computer and Communications (ICCC), 7-10 Dec. 2018. pp. 2002-2006. DOI: https://doi. org/10.1109/CompComm.2018.8780772

33. Andrei $\mathbf{D}$, et al. Computer aided patient evaluation in the low back pain pathology. In SACI 2015 - 10th Jubilee IEEE International Symposium on Applied Computational Intelligence and Informatics, Proceedings, 2015. pp. 27-30. [Online]. Available: https://www.scopus.com/inward/ record. uri?eid=2-s2.0-84959288395\&doi=10.1109\%2fs ACI.2015.7208233\&partnerID $=40 \& \mathrm{md} 5=036 \mathrm{bf} 4 \mathrm{a} 4 \mathrm{~b} 7 \mathrm{~d} 8$ ee995a7d1012de69826f. DOI: https://doi.org/10.1109/ SACI.2015.7208233

34 Olugbade TA, Aung MSH, Bianchi-Berthouze N, Marquardt N, Williams AC. Bi-Modal detection of painful reaching for chronic pain rehabilitation systems. In ICMI 2014 - Proceedings of the 2014 International Conference on Multimodal Interaction, 2014. pp. 455-458. [Online]. Available: https://www.scopus.com/inward/record.uri?eid=2 s2.0-84947232471\&doi=10.1145\%2f2663204.2663261\&p artnerID $=40 \& \mathrm{md} 5=\mathrm{e} 25 f 5 \mathrm{dd} 959536 \mathrm{af701} \mathrm{e} 3 \mathrm{c} 970 \mathrm{e} 368 \mathrm{f} 60 \mathrm{~d}$. DOI: https://doi.org/10.1145/2663204.266326

35. Olugbade TA, Bianchi-Berthouze N, Marquardt N, Williams AC, Ieee. Pain Level Recognition using Kinematics and Muscle Activity for Physical Rehabilitation in Chronic Pain. In 2015 International Conference on Affective Computing and Intelligent Interaction, (International Conference on Affective Computing and Intelligent Interaction, 2015. pp. 243-249. DOI: https://doi. org/10.1109/ACII.2015.7344578

36. Rabbi M, Aung MS, Gay G, Reid MC, Choudhury T. Feasibility and Acceptability of Mobile Phone-Based AutoPersonalized Physical Activity Recommendations for Chronic Pain Self-Management: Pilot Study on Adults (in eng). J. Med. Internet Res. Oct 26 2018; 20(10): e10147. DOI: https://doi.org/10.2196/10147

37. Abdollahi M, et al. Using a Motion Sensor to Categorize Nonspecific Low Back Pain Patients: A Machine Learning Approach (in eng). Sensors (Basel). Jun 26 2020; 20(12). DOI: https://doi.org/10.3390/s20123600

38. Tuechler K, Fehrmann E, Kienbacher T, Mair P, Fischer-Grote L, Ebenbichler G. Mapping patient reported outcome measures for low back pain to the International Classification of Functioning, Disability and Health using random forests (in eng). Eur. J. Phys. Rehabil. Med. Jun 2020; 56(3): 286-296. DOI: https://doi.org/10.23736/S19739087.20.05465-9

39. Liew BXW, Rugamer D, De Nunzio AM, Falla D. Interpretable machine learning models for classifying low back pain status using functional physiological variables (in eng). Eur. Spine J. Aug 2020; 29(8): 1845-1859. DOI: https:// doi.org/10.1007/s00586-020-06356-0

40. Gal N, Stoicu-Tivadar V, Andrei D, Nemeş DI, Nǎdǎşan

E. Computer assisted treatment prediction of low 
back pain pathologies. Studies in Health Technology and Informatics. 2014; 197: 47-51. [Online]. Available: https://www.scopus.com/inward/record.uri?eid=2-s2.084903714766\&doi=10.3233\%2f978-1-61499-389-6-47\&par

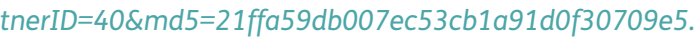
DOI: https://doi.org/10.3233/978-1-61499-389-6-47

41 Aung MSH, Bianchi-Berthouze N, Watson P, De C. Williams AC. Automatic recognition of fear-avoidance behavior in chronic pain physical rehabilitation. In Proceedings - PERVASIVEHEALTH 2014: 8th International Conference on Pervasive Computing Technologies for Healthcare, 2014. pp. 158-161. [Online]. Available: https://www.scopus.com/inward/record. uri?eid=2-s2.0-84928911378\&doi=10.4108\%2ficst. pervasivehealth.2014.254945\&partner ID $=40 \& m d 5=1$ aedaad27b37d68a2e5cda54b373ad6a. DOI: https://doi.org/10.4108/icst.pervasivehealth.2014.254945

42. Haider F, Albert P, Luz S. Automatic Recognition of Low-Back Chronic Pain Level and Protective Movement Behaviour using Physical and Muscle Activity Information. In 2020 15th IEEE International Conference on Automatic Face and Gesture Recognition (FG 2020), 16-20 Nov. 2020. pp. 834-838. DOI: https://doi.org/10.1109/ FG47880.2020.00065

43. Payne KB, Wharrad H, Watts K. Smartphone and medical related App use among medical students and junior doctors in the United Kingdom (UK): a regional survey (in eng). BMC Med. Inform. Decis. Mak. Oct 30 2012; 12: 121. DOI: https://doi.org/10.1186/1472-6947-12-121

44. Rabbi M, Aung MSH, Gay G, Reid MC, Choudhury T. Feasibility and acceptability of mobile phone-based auto-personalized physical activity recommendations for chronic pain self-management: Pilot study on adults. J. Med. Internet Res. 2018; 20(10): e10147. DOI: https://doi. org/10.2196/10147

45. Rathbone AL, Clarry L, Prescott J. Assessing the Efficacy of Mobile Health Apps Using the Basic Principles of Cognitive
Behavioral Therapy: Systematic Review (in eng). J. Med. Internet Res. 2017; 19(11): e399-e399. DOI: https://doi. org/10.2196/jmir.8598

46. Zale EL, Ditre JW. Pain-Related Fear, Disability, and the Fear-Avoidance Model of Chronic Pain (in eng). Curr Opin Psychol. 2015; 5: 24-30. DOI: https://doi.org/10.1016/j. copsyc.2015.03.014

47. Stucki G, Cieza A, Melvin J. The International Classification of Functioning, Disability and Health (ICF): a unifying model for the conceptual description of the rehabilitation strategy (in eng). J. Rehabil. Med. 2007; 39(4): 279-285. DOI: https:// doi.org/10.2340/16501977-0041

48. Silva L, Vaz JR, Castro MA, Serranho P, Cabri J, Pezarat-Correia P. Recurrence quantification analysis and support vector machines for golf handicap and low back pain EMG classification (in eng) J. Electromyogr. Kinesiol. Aug 2015; 25(4): 637-47. DOI: https://doi.org/10.1016/j. jelekin.2015.04.008

49. Jubany J, Danneels L, Angulo-Barroso R. The influence of fatigue and chronic low back pain on muscle recruitment patterns following an unexpected external perturbation (in eng). BMC Musculoskelet. Disord. 2017; 18(1): 161-161. DOI: https://doi.org/10.1186/s12891-017-1523-3

50. Higgins JP, et al. The Cochrane Collaboration's tool for assessing risk of bias in randomised trials (in eng). Bmj. Oct 18 2011; 343: d5928. DOI: https://doi.org/10.1136/bmj. d5928

51. Sterne JA, et al. ROBINS-I: a tool for assessing risk of bias in non-randomised studies of interventions (in eng). Bmj. Oct 12 2016; 355: i4919. DOI: https://doi.org/10.1136/bmj.i4919

52. Eldridge SM, et al. CONSORT 2010 statement: extension to randomised pilot and feasibility trials. BMJ. 2016; 355: i5239. DOI: https://doi.org/10.1136/bmj.i5239

53. Neugebauer EAM, et al. Specific barriers to the conduct of randomised clinical trials on medical devices (in eng). Trials. 2017; 18(1): 427-427. DOI: https://doi.org/10.1186/s13063017-2168-0

TO CITE THIS ARTICLE:

Amorim P, Paulo JR, Silva PA, Peixoto P, Castelo-Branco M, Martins H. Machine Learning Applied to Low Back Pain Rehabilitation - A Systematic Review. International Journal of Digital Health. 2021; 1(1): 10, 1-14. DOI: https://doi.org/10.29337/ijdh.34

Submitted: 29 January $2021 \quad$ Accepted: 25 March $2021 \quad$ Published: 29 April 2021

COPYRIGHT:

(c) 2021 The Author(s). This is an open-access article distributed under the terms of the Creative Commons AttributionNonCommercial 4.0 International License (CC-BY-NC 4.0), which permits unrestricted distribution, reproduction and adaptation in any medium, provided the original author and source are credited, and that the material is not used for commercial purposes. See https://creativecommons.org/licenses/by-nc/4.0/.

International Journal of Digital Health is a peer-reviewed open access journal published by IJS Publishing Group. 